\title{
A position-dependent mass harmonic oscillator and deformed space
}

\author{
Bruno G. da Costa* \\ Instituto Federal de Educação, Ciência e Tecnologia do Sertão Pernambucano, \\ Campus Petrolina, BR 407, $\mathrm{km} \mathrm{08,}$ \\ 56314-520 Petrolina, Pernambuco, Brazil and \\ Instituto de Física, Universidade Federal da Bahia, \\ Rua Barao de Jeremoabo, 40170-115 Salvador-BA, Brasil \\ Ernesto P. Borged \\ Instituto de Física, Universidade Federal da Bahia, \\ Rua Barao de Jeremoabo, 40170-115 Salvador-BA, Brasil and \\ National Institute of Science and Technology for Complex Systems, \\ Rua Xavier Sigaud 150, Rio de Janeiro 22290-180, Brazil
}

(Dated: March 28, 2018) 


\begin{abstract}
We consider canonically conjugated generalized space and linear momentum operators $\hat{x}_{q}$ and $\hat{p}_{q}$ in quantum mechanics, associated to a generalized translation operator which produces infinitesimal deformed displacements controlled by a deformation parameter $q$. A canonical transformation $(\hat{x}, \hat{p}) \rightarrow\left(\hat{x}_{q}, \hat{p}_{q}\right)$ leads the Hamiltonian of a position-dependent mass particle in usual space to another Hamiltonian of a particle with constant mass in a conservative force field of the deformed space. The equation of motion for the classical phase space $(x, p)$ may be expressed in terms of the deformed (dual) q-derivative. We revisit the problem of a $q$-deformed oscillator in both classical and quantum formalisms. Particularly, this canonical transformation leads a particle with positiondependent mass in a harmonic potential to a particle with constant mass in a Morse potential. The trajectories in phase spaces $(x, p)$ and $\left(x_{q}, p_{q}\right)$ are analyzed for different values of the deformation parameter. Lastly, we compare the results of the problem in classical and quantum formalisms through the principle of correspondence and the WKB approximation.
\end{abstract}

PACS numbers: 03.65.Ca, 03.65.Ge, 03.65.Sq

\footnotetext{
* bruno.costa@ifsertao-pe.edu.br

$\dagger$ ernesto@ufba.br
} 


\section{INTRODUCTION}

During last three decades experimental and theoretical studies of quantum systems with position-dependent mass (PDM) has attracted the interest of several researchers of different areas. The most common example of an application of such systems is the motion of electrons and holes in semiconductors [1]. There are several others applications of quantum systems with PDM, for instance, studies on inversion potential for $\mathrm{NH}_{3}$ in density functional theory [2], He clusters [3], optical properties of a spherical quantum dot [4], neutrino mass oscillations [5].

In many theoretical works related to systems with PDM, the main goal is to obtain the eigenfunctions and energy levels by means of the Schrödinger equation for a system with a certain function of the mass in terms of the position and subject to a specific potential. Generally, the Schrödinger equation for a system with PDM is a non-homogeneous differential equation. The most common approaches used to solve systems with PDM are coordinate transformations and supersymmetry [6, 7].

Recently, Costa Filho et al. have proposed a generalized translation operator which produces infinitesimal nonlinear displacements, defined by [8-11]

$$
\hat{\mathcal{T}}_{q}(\varepsilon)|x\rangle \equiv\left|x+\varepsilon+\gamma_{q} x \varepsilon\right\rangle .
$$

It may be associated with the $q$-algebra derived from the nonextensive formalism through $\gamma_{q} \equiv(1-q) / \xi[12,13]$. The index $q$ is a dimensionless parameter, and $\xi$ is a characteristic length. The operator $\hat{\mathcal{T}}_{q}(\varepsilon)$ leads to a generator of spatial translations corresponding to a position-dependent linear momentum given by

$$
\hat{p}_{q}^{\prime}=\left(\hat{1}+\gamma_{q} \hat{x}\right) \hat{p}
$$

and consequently a particle with PDM. This deformed momentum operator has been used to discuss a system with PDM for different potentials in the quantum formalism [8 11,14 , 20] . In these works, the deformed linear momentum operator is not Hermitian at the coordinate basis $\{|x\rangle\}$. A modification can be done in order to make it Hermitian [10, 11, 17, 18]:

$$
\hat{p}_{q}=\frac{\left(\hat{1}+\gamma_{q} \hat{x}\right) \hat{p}}{2}+\frac{\hat{p}\left(\hat{1}+\gamma_{q} \hat{x}\right)}{2}=\left(\hat{1}+\gamma_{q} \hat{x}\right) \hat{p}-\frac{i \hbar \gamma_{q}}{2},
$$

with $[\hat{x}, \hat{p}]=i \hbar \hat{1}$. Hermiticity leads to important properties: classical analogues for operators of dynamic variables, real eigenvalues, and the existence of orthonormal basis of eigenstates. This latter is related to the conservation law for probability in quantum mechanics. 
Nonlinear generalizations of equations of motion formulated by means of the $q$-algebra have been recently proposed [21 28]. Alternative generalizations of dynamic equations with Jackson's q-calculus can be found in the literature [29-33]. A classical deformed oscillator described by deformed trigonometric functions has been introduced in [34]. Ref. [9] analyzed a quantum harmonic oscillator in a nonlinear space given by Eq. (11), with the non-Hermitian operator (2). Deformed harmonic oscillators have nonlinear spectrum and can describe diatomic molecules. One important drawback of these formulations is the violation of the uncertainty principle for certain values of the controlling parameter.

We revisit the problem of a particle in a nonlinear space as described by Eq. (11), and its linear momentum given by Eq. (3) under the influence of the quadratic potential $V(x)=$ $k x^{2} / 2$, within both classical and quantum formalisms. The paper is organized as follows: we briefly review some properties of the $q$-algebra. Next, we discuss the dynamics of a system in the nonlinear space for both classical and quantum formalisms. Then, we solve the problem of a particle with PDM in classical and quantum formalisms, comparing the results through the WKB approximation and the principle of correspondence. Uncertainty principle is also analyzed.

\section{DEFORMED FUNCTIONS AND DERIVATIVES}

The $q$-exponential is a generalization of the ordinary exponential function, defined by:

$$
\exp _{q} x \equiv[1+(1-q) x]_{+}^{1 /(1-q)}
$$

with $[A]_{+}=\max \{A, 0\}$, and the ordinary exponential is recovered as $q \rightarrow 1$ [35-37]. It satisfies $\exp _{q}(a) \exp _{q}(b)=\exp _{q}\left(a \oplus_{q} b\right)$ and $\exp _{q}(a) / \exp _{q}(b)=\exp _{q}\left(a \ominus_{q} b\right)$, where the symbol $\oplus_{q}$ represents the $q$-addition operator defined by $a \oplus_{q} b \equiv a+b+(1-q) a b$, and $\ominus_{q}$ represents the $q$-subtraction, $a \ominus_{q} b \equiv \frac{a-b}{1+(1-q) b} \quad\left(b \neq(q-1)^{-1}\right)$ [12, 13]. The inverse function of the $q$-exponential is the $q$-logarithm function, given by

$$
\ln _{q} x \equiv \frac{x^{1-q}-1}{1-q} \quad(x>0)
$$

It is possible to define a generalization of the derivative operator, based on these deformed 
algebraic operators [13]. Particularly,

$$
\begin{aligned}
D_{q} f(u) & \equiv \lim _{u^{\prime} \rightarrow u} \frac{f\left(u^{\prime}\right)-f(u)}{u^{\prime} \ominus_{q} u} \\
& =\lim _{\Delta u \rightarrow 0} \frac{f\left(u \oplus_{q} \Delta u\right)-f(u)}{\Delta u} \\
& =[1+(1-q) u] \frac{d f(u)}{d u},
\end{aligned}
$$

which the $q$-exponential is eigenfunction. There is a dual $q$-derivative,

$$
\begin{aligned}
\widetilde{D}_{q} f(u) & \equiv \lim _{u^{\prime} \rightarrow u} \frac{f\left(u^{\prime}\right) \ominus_{q} f(u)}{u^{\prime}-u} \\
& =\frac{1}{1+(1-q) f(u)} \frac{d f(u)}{d u},
\end{aligned}
$$

that satisfies $\widetilde{D}_{q} \ln _{q} u=1 / u$. These operators obey $\widetilde{D}_{q} x(y)=\left[D_{q} y(x)\right]^{-1}, \forall q \in \mathbb{R}$. The ordinary derivative is recovered for $q=1$ in both cases.

The deformed derivative operator $D_{q} f(u)$ may be understood as the rate of variation of the function $f(u)$ with respect to a nonlinear variation of the independent variable $u \rightarrow u^{\prime}=$ $u \oplus_{q} \Delta u=u+\Delta u+(1-q) u \Delta u$. On the other hand, the dual deformed derivative operator $\widetilde{D}_{q} f(u)$ is the rate of a nonlinear variation of the function $f(u)$ with respect to the ordinary variation of the independent variable $u$.

Considering a real variable $u$, we have

$$
d_{q} u \equiv \lim _{u^{\prime} \rightarrow u} u^{\prime} \ominus_{q} u=\frac{d u}{1+(1-q) u} .
$$

The definition of the deformed variable $u_{q}$ (a deformed $q$-number)

$$
u_{q} \equiv \ln \left(\exp _{q} u\right)=\frac{\ln [1+(1-q) u]}{(1-q)}
$$

implies $d_{q} u=d u_{q}$, i.e., the deformed differential of an ordinary variable $u$ may be expressed as the ordinary differential of a deformed variable $u_{q}$. This $q$-deformed variable, Eq. (91), has already been defined in [34]. It is curious to notice that Tsallis an Rényi entropies are related exactly through this transformation (see Eq. (8) of [36]).

The $q$-derivatives obey the following relations: $D_{q} f(u)=d f(u) / d_{q} u=d f(u) / d u_{q}$ and $\widetilde{D}_{q} f(u)=d_{q} f(u) / d u=d f_{q}(u) / d u$, i.e., the deformed derivative of an ordinary variable is equal to the ordinary derivative of the corresponding deformed variable. The second $q$-derivatives must be used as

$$
D_{q}^{2} f(u)=[1+(1-q) u] \frac{d}{d u}\left\{[1+(1-q) u] \frac{d f}{d u}\right\},
$$


and

$$
\widetilde{D}_{q}^{2} f(u)=\frac{1}{1+(1-q) f(u)} \frac{d}{d u}\left[\frac{1}{1+(1-q) f(u)} \frac{d f}{d u}\right] .
$$

Higher order deformed derivatives are evaluated accordingly.

\section{DYNAMICS OF A SYSTEM WITH POSITION-DEPENDENT MASS}

The deformed linear momentum operator (2) is a generator of nonlinear translations. This operator is related to the $q$-derivative (6) . The effect of operator $\hat{p}_{q}^{\prime}$ at coordinate basis $\{|x\rangle\}$ on a state $|\alpha\rangle$ is

$$
\left\langle x\left|\hat{p}_{q}^{\prime}\right| \alpha\right\rangle=-i \hbar\left(1+\gamma_{q} x\right) \frac{d \psi(x)}{d x}=-i \hbar D_{\gamma_{q}} \psi(x),
$$

where $\psi(x)=\langle x \mid \alpha\rangle$ and $D_{\gamma_{q}} \equiv\left(1+\gamma_{q} x\right) d / d x$. Similarly,

$$
\begin{aligned}
\left\langle x\left|\hat{p}_{q}\right| \alpha\right\rangle & =-i \hbar\left(1+\gamma_{q} x\right) \frac{d \psi(x)}{d x}-\frac{i \hbar \gamma_{q}}{2} \psi(x) \\
& =-i \hbar D_{\gamma_{q}} \psi(x)-\frac{i \hbar \gamma_{q}}{2} \psi(x) .
\end{aligned}
$$

Using the property $[\hat{p}, f(\hat{x})]=-i \hbar d f(\hat{x}) / d \hat{x}$ with $f(\hat{x})=\left(\hat{1}+\gamma_{q} \hat{x}\right)^{1 / 2}$, we can also write $\hat{p}_{q}=\left(\hat{1}+\gamma_{q} \hat{x}\right)^{1 / 2} \hat{p}\left(\hat{1}+\gamma_{q} \hat{x}\right)^{1 / 2}$. Thus,

$$
\left\langle x\left|\hat{p}_{q}\right| \alpha\right\rangle=\sqrt{1+\gamma_{q} x}\left(\frac{\hbar}{i} \frac{d}{d x}\right)\left[\sqrt{1+\gamma_{q} x} \psi(x)\right] .
$$

Introducing a generalized space operator $\hat{x}_{q}$ canonically conjugated to $\hat{p}_{q}$, i.e. $\left[\hat{x}_{q}, \hat{p}_{q}\right]=$ $i \hbar \hat{1}$, we get

$$
\hat{x}_{q}=\frac{\ln \left(\hat{1}+\gamma_{q} \hat{x}\right)}{\gamma_{q}}=\xi \ln \left[\exp _{q}(\hat{x} / \xi)\right] .
$$

In particular, $(\hat{x}, \hat{p}) \longrightarrow\left(\hat{x}_{q}, \hat{p}_{q}\right)$ forms a canonical transformation that leads a $q$-addition of two positions at basis $\{|x\rangle\}$ into a usual addition of two positions in a deformed space at basis $\left\{\left|x_{q}\right\rangle\right\}$, i.e., $x^{\prime} \oplus_{q} x \longrightarrow x_{q}^{\prime}+x_{q}$. These hermitian operators present the following classical analogs:

$$
\left\{\begin{array}{l}
p_{q}=\left(1+\gamma_{q} x\right) p \\
x_{q}=\frac{\ln \left(1+\gamma_{q} x\right)}{\gamma_{q}}=\xi \ln \left[\exp _{q}(x / \xi)\right]
\end{array}\right.
$$

with the generating function given by $\phi\left(x_{q}, p\right)=-p\left(e^{\gamma_{q} x_{q}}+1\right) / \gamma_{q}$. In the following, we analyze some implications of this canonical transformation in both classical and quantum formalisms. 


\section{A. Deformed classical formalism}

Let us initially address the classical problem of a constant mass particle submitted to a conservative force with potential $V\left(x_{q}\right)$, and the linear deformed momentum $p_{q}$, whose Hamiltonian is

$$
K\left(x_{q}, p_{q}\right)=\frac{p_{q}^{2}}{2 m_{0}}+V\left(x_{q}\right)
$$

The canonical transformation given by Eqs. (16) leads to the new Hamiltonian (see, for instance, [38])

$$
H(x, p)=\frac{p^{2}}{2 m(x)}+V(x)
$$

with

$$
m(x)=\frac{m_{0}}{\left(1+\gamma_{q} x\right)^{2}}
$$

The equation of motion is

$$
\dot{p}=-\frac{\gamma_{q}\left(1+\gamma_{q} x\right) p^{2}}{m_{0}}-\frac{d V(x)}{d x},
$$

with $p=m(x) \dot{x}$, thus

$$
m_{0}\left[\frac{\ddot{x}}{\left(1+\gamma_{q} x\right)^{2}}-\frac{\gamma_{q} \dot{x}^{2}}{\left(1+\gamma_{q} x\right)^{3}}\right]=-\frac{d V(x)}{d x} .
$$

This equation may be conveniently rewritten as

$$
m_{0} \widetilde{D}_{\gamma_{q}}^{2} x(t)=F(x)
$$

i.e., a deformed Newton's law for a space with nonlinear displacements. The generalized displacement of a PDM $m(x)$ in a usual space $\left(d_{q} x\right)$ is mapped into a constant mass $m_{0}$ in a deformed space with usual displacement $\left(d x_{q}\right): d_{q} x \equiv \xi\left[\left(\frac{x+d x}{\xi}\right) \ominus_{q}\left(\frac{x}{\xi}\right)\right]=\frac{d x}{1+\gamma_{q} x} \equiv$ $d x_{q}$. The time evolution is, thus, governed by the generalized dual derivative, $\widetilde{D}_{\gamma_{q}} x=$ $\frac{1}{1+\gamma_{q} x} \frac{d x}{d t}$.

The particle velocity,

$$
\dot{x}=\sqrt{\frac{2}{m(x)}[E-V(x)]},
$$

may be rewritten as a deformed particle velocity:

$$
\widetilde{D}_{\gamma_{q}} x=\sqrt{\frac{2}{m_{0}}[E-V(x)]} .
$$


Coherently, the particle position can be obtained through the $q$-integral

$$
\begin{aligned}
t-t_{0} & = \pm \int_{x_{0}}^{x} \frac{d x}{\sqrt{\frac{2}{m(x)}[E-V(x)]}} \\
& = \pm \int_{x_{0}}^{x} \frac{d x}{\left(1+\gamma_{q} x\right) \sqrt{\frac{2}{m_{0}}[E-V(x)]}} \\
& = \pm \int_{x_{0}}^{x} \frac{d_{q} x}{\sqrt{\frac{2}{m_{0}}[E-V(x)]}}
\end{aligned}
$$

\section{B. Deformed quantum formalism}

Consider a quantum system described by the Hamiltonian $\hat{K}\left(\hat{x}_{q}, \hat{p}_{q}\right)=\hat{p}_{q}^{2} / 2 m_{0}+V\left(\hat{x}_{q}\right)$, at the coordinate basis $\left\{\left|x_{q}\right\rangle\right\}$. For the ket state $|\alpha(t)\rangle$, the Schrödinger equation is $i \hbar \frac{\partial}{\partial t}|\alpha(t)\rangle=$ $\hat{K}|\alpha(t)\rangle$, i.e.

$$
i \hbar \frac{\partial \Phi\left(x_{q}, t\right)}{\partial t}=-\frac{\hbar^{2}}{2 m_{0}} \frac{\partial^{2} \Phi\left(x_{q}, t\right)}{\partial x_{q}^{2}}+V\left(x_{q}\right) \Phi\left(x_{q}, t\right)
$$

with $\Phi\left(x_{q}, t\right) \equiv\left\langle x_{q} \mid \alpha(t)\right\rangle$.

From the canonical transformation given by Eqs. (3) and (15), the Hamiltonian operator at basis $\{|x\rangle\}$ is

$$
\begin{aligned}
\hat{H}(\hat{x}, \hat{p}) & =\frac{1}{2 m_{0}}\left[\frac{\left(\hat{1}+\gamma_{q} \hat{x}\right) \hat{p}}{2}+\frac{\hat{p}\left(\hat{1}+\gamma_{q} \hat{x}\right)}{2}\right]^{2}+V(\hat{x}) \\
& =\frac{1}{2 m_{0}}\left[\left(\hat{1}+\gamma_{q} \hat{x}\right)^{1 / 2} \hat{p}\left(\hat{1}+\gamma_{q} \hat{x}\right) \hat{p}\left(\hat{1}+\gamma_{q} \hat{x}\right)^{1 / 2}\right]+V(\hat{x}) .
\end{aligned}
$$

It is in agreement with kinetic operator introduced by von Roos [1] for systems with a PDM operator given by $m(\hat{x})=m_{0} /\left(\hat{1}+\gamma_{q} \hat{x}\right)^{2}$, and it can be rewritten as

$$
\hat{H}(\hat{x}, \hat{p})=-\frac{\hbar^{2}}{2}\left\{[m(\hat{x})]^{\varsigma} \frac{d}{d x}[m(\hat{x})]^{\zeta} \frac{d}{d x}[m(\hat{x})]^{\varsigma}\right\}+V(\hat{x})
$$

with $\varsigma=\frac{\zeta}{2}=-\frac{1}{4}$. The particular case $(\varsigma, \zeta)=\left(-\frac{1}{4},-\frac{1}{2}\right)$ maps the potential $V(\hat{x})$ into an effective potential $V\left(\hat{x}_{q}\right)$, that is independent on $m(\hat{x})$ [39].

The Schrödinger equation $i \hbar \frac{\partial}{\partial t}|\alpha(t)\rangle=\hat{H}|\alpha(t)\rangle$ may be explicitly written in terms of the wave function $\Psi(x, t) \equiv\langle x \mid \alpha(t)\rangle$ as

$i \hbar \frac{\partial \Psi(x, t)}{\partial t}=-\frac{\hbar^{2}\left(1+\gamma_{q} x\right)^{2}}{2 m_{0}} \frac{\partial^{2} \Psi(x, t)}{\partial x^{2}}-\frac{\hbar^{2} \gamma_{q}\left(1+\gamma_{q} x\right)}{m_{0}} \frac{\partial \Psi(x, t)}{\partial x}-\frac{\hbar^{2} \gamma_{q}^{2}}{8 m_{0}} \Psi(x, t)+V(x) \Psi(x, t)$. 
The probability density $\rho(x, t) \equiv|\Psi(x, t)|^{2}$ obeys the continuity equation

$$
\frac{\partial \rho(x, t)}{\partial t}+\frac{\partial J(x, t)}{\partial x}=0
$$

where the current density is given by

$$
J(x, t) \equiv \operatorname{Re}\left\{\Psi^{*}(x, t)\left(\frac{\hbar}{i} \frac{\partial}{\partial x}\right)\left[\frac{1}{m(x)} \Psi(x, t)\right]\right\}
$$

Eq. (29) may be conveniently rewritten by means of the transformation commonly use for PDM systems, $\Psi(x, t)=\left[m(x) / m_{0}\right]^{1 / 4} \Phi_{q}(x, t)[39]$, that results

$$
\Psi(x, t) \equiv \frac{\Phi_{q}(x, t)}{\sqrt{1+\gamma_{q} x}},
$$

and

$$
i \hbar \frac{\partial \Phi_{q}(x, t)}{\partial t}=-\frac{\hbar^{2}\left(1+\gamma_{q} x\right)^{2}}{2 m_{0}} \frac{\partial^{2} \Phi_{q}(x, t)}{\partial x^{2}}-\frac{\hbar^{2} \gamma_{q}\left(1+\gamma_{q} x\right)}{2 m_{0}} \frac{\partial \Phi_{q}(x, t)}{\partial x}+V(x) \Phi_{q}(x, t),
$$

or, more compactly,

$$
i \hbar \frac{\partial \Phi_{q}(x, t)}{\partial t}=-\frac{\hbar^{2}}{2 m_{0}} \mathcal{D}_{\gamma_{q}}^{2} \Phi_{q}(x, t)+V(x) \Phi_{q}(x, t) .
$$

This equation is equivalent to Eq. (26) with $x \longrightarrow x_{q}=\xi \ln \left[\exp _{q}(x / \xi)\right], \mathcal{D}_{\gamma_{q}} \equiv \partial / \partial x_{q}=(1+$ $\left.\gamma_{q} x\right) \partial / \partial x$, and $\Phi_{q}(x, t)=\Phi\left(x_{q}(x), t\right)$. It is also equivalent to Eq. (14) of [8] which corresponds to a Schrödinger-like equation for a field $\Phi_{q}(x, t)$ with the non-hermitian operator $\hat{p}_{q}^{\prime}$, and an associated non-Hermitian Hamiltonian operator given by

$$
\begin{aligned}
\hat{H}^{\prime} & =\frac{1}{2 m_{0}}\left(\hat{p}_{q}^{\prime}\right)^{2}+V(\hat{x}) \\
& =\frac{1}{2 m_{0}}\left(1+\gamma_{q} \hat{x}\right) \hat{p}\left(1+\gamma_{q} \hat{x}\right) \hat{p}+V(\hat{x}) .
\end{aligned}
$$

There is an equivalence between describing a Hermitian system with kinetic energy term of a PDM and a non-Hermitian one with kinetic energy term deformed in terms of the spatial $q$-derivative. Furthermore, the field $\Psi(x, t)$ is replaced by a new deformed field $\Phi_{q}(x, t)$. Similar to the classical formalism, the Schrödinger-like equation (34) for a system with PDM may also be written in terms of the $q$-derivative.

Note that if the field $\Phi\left(x_{q}, t\right)$ is the solution of the Schrödinger equation at basis $\left\{\left|x_{q}\right\rangle\right\}$, then the field $\Psi(x, t)$ is its solution at basis $\{|x\rangle\}$. Thus, the Schrödinger equation for the field $\Psi(x, t)$ for a system with PDM in a usual space $\{|x\rangle\}$ is mapped into an equation for 
the field $\Phi\left(x_{q}, t\right)$ in a deformed space $\left\{\left|x_{q}\right\rangle\right\}$. Furthermore, if $\Phi\left(x_{q}, t\right)$ is normalized, then $\Psi(x, t)$ is also normalized. In fact,

$$
\int_{x_{q, i}}^{x_{q, f}} \Phi^{*}\left(x_{q}, t\right) \Phi\left(x_{q}, t\right) d x_{q}=\int_{x_{i}}^{x_{f}} \frac{\Phi_{q}^{*}(x, t) \Phi_{q}(x, t)}{1+\gamma_{q} x} d x=1
$$

and using Eq. (32), we have $\int \Psi^{*}(x, t) \Psi(x, t) d x=1$. The deformed space implies a deformed metric, with the deformed inner product defined by the $q$-integral (see [9]):

$$
\begin{aligned}
\left\langle\varphi_{b}(x) \mid \varphi_{a}(x)\right\rangle_{q} & \equiv \int_{x_{i}}^{x_{f}} \frac{\varphi_{b}^{*}(x) \varphi_{a}(x)}{1+\gamma_{q} x} d x \\
& =\int_{x_{i}}^{x_{f}} \varphi_{b}^{*}(x) \varphi_{a}(x) d_{q} x \\
& =\int_{x_{q, i}}^{x_{q, f}} \varphi_{b}^{*}\left(x_{q}\right) \varphi_{a}\left(x_{q}\right) d x_{q} \\
& =\left\langle\varphi_{b}\left(x_{q}\right) \mid \varphi_{a}\left(x_{q}\right)\right\rangle .
\end{aligned}
$$

The deformed continuity equation reads:

$$
\frac{\partial \varrho_{q}(x, t)}{\partial t}+\mathcal{D}_{\gamma_{q}} \mathcal{J}_{q}(x, t)=0
$$

with

$$
\mathcal{J}_{q}(x, t) \equiv \operatorname{Re}\left[\Phi_{q}^{*}(x, t)\left(\frac{\hbar}{i} \mathcal{D}_{\gamma_{q}}\right)\left(\frac{\Phi_{q}(x, t)}{m_{0}}\right)\right] .
$$

Consistently, Eqs. (30)-(32) lead to (38)-(39) .

The quantum formalism for PDM in terms of the field $\Phi_{q}(x, t)$ replaces the usual derivative and integral operators (with respect to the spatial variable $x$ ) by the $q$-derivative and $q$ integral. The same feature applies for the classical formalism, but the with the dual $q$ derivative, instead. This is due to the fact that in the quantum formalism, the equations that describe the dynamics of the system (such as the Schrödinger equation), takes into account nonlinear spatial variations of the independent variable $x\left(\Phi=\Phi_{q}(x, t)\right)$, which is directly related to the $q$-derivative (66). On the contrary, in the classical formalism, the nonlinear spatial variation takes place on the dependent variable $x(t)$, directly associated with the definition of the dual $q$-derivative (7).

According to Ehrenfest's theorem, the time evolution of the expectation values of the space $\hat{x}$ and linear momentum $\hat{p}$ operators are respectively given by

$$
\frac{d\langle\hat{x}\rangle}{d t}=\frac{\left\langle\left(\hat{1}+\gamma_{q} \hat{x}\right) \hat{p}\left(\hat{1}+\gamma_{q} \hat{x}\right)\right\rangle}{m_{0}}
$$


and

$$
\frac{d\langle\hat{p}\rangle}{d t}=-\frac{\gamma_{q}}{m_{0}}\left\langle\hat{p}\left(\hat{1}+\gamma_{q} \hat{x}\right) \hat{p}\right\rangle-\left\langle\frac{d V}{d \hat{x}}\right\rangle,
$$

with $\int J(x, t) d x=d\langle\hat{x}\rangle / d t$.

\section{CLASSICAL HARMONIC OSCILLATOR WITH POSITION-DEPENDENT MASS}

Consider a particle with mass given by Eq. (19) under the influence of a quadratic potential, whose the Hamiltonian is

$$
H(x, p)=\frac{p^{2}}{2 m(x)}+\frac{1}{2} k x^{2} .
$$

The deformed Newton's law for this problem is:

$$
\widetilde{D}_{\gamma_{q}}^{2} x(t)=-\omega_{0}^{2} x,
$$

where the angular frequency $\omega_{0}=\sqrt{k / m_{0}}$ corresponds to the usual case $\gamma_{q}=0$.

The velocity, $v(t)=\dot{x}= \pm\left(1+\gamma_{q} x\right) \omega_{0} \sqrt{A^{2}-x^{2}}$, is rewritten as

$$
\widetilde{D}_{\gamma_{q}} x(t)= \pm \omega_{0} \sqrt{A^{2}-x^{2}}
$$

where $A$ is the amplitude of oscillations. The solution of Eq. (42), or Eq. (43), is

$$
x(t)=A \cos \left[\theta_{q}(t)\right]
$$

with

$$
\theta_{q}(t)=2 \operatorname{atan}\left[\sqrt{\frac{1+\gamma_{q} A}{1-\gamma_{q} A}} \tan \left(\sqrt{1-\gamma_{q}^{2} A^{2}} \frac{\omega_{0} t+\delta}{2}\right)\right],
$$

that is a periodic function with period $\tau_{q}=\frac{2 \pi}{\omega_{0} \sqrt{1-\gamma_{q}^{2} A^{2}}}$. Figure 1 shows position, velocity, acceleration and phase for $0 \leq \gamma_{q} A<1$ (the usual case $\gamma_{q} A=0$ is illustrated for comparison). As $\gamma_{q} A$ approaches 1 , the particle remains close to the position $x=-A$ for longer periods of time, since the mass is increased in this region.

For $\gamma_{q} A>1$, the system looses its oscillatory nature and the particle moves between $-1 / \gamma_{q}<x \leq A$, as illustrated in Figure 2. When $t \gg \tau_{0}, x$ asymptotically approaches $x_{\min }=-1 / \gamma_{q}$. Figure 2 also shows the particle velocity as a function of time. For $t>t^{*}$, where

$$
t^{*}=\frac{\tau_{q}}{\pi} \operatorname{atanh}\left[\frac{\left(\gamma_{q} A-1\right)\left(1+4 \gamma_{q} A-\sqrt{1+8 \gamma_{q}^{2} A^{2}}\right)}{\left(\gamma_{q} A+1\right)\left(1+4 \gamma_{q} A+\sqrt{1+8 \gamma_{q}^{2} A^{2}}\right)}\right]^{1 / 2}
$$



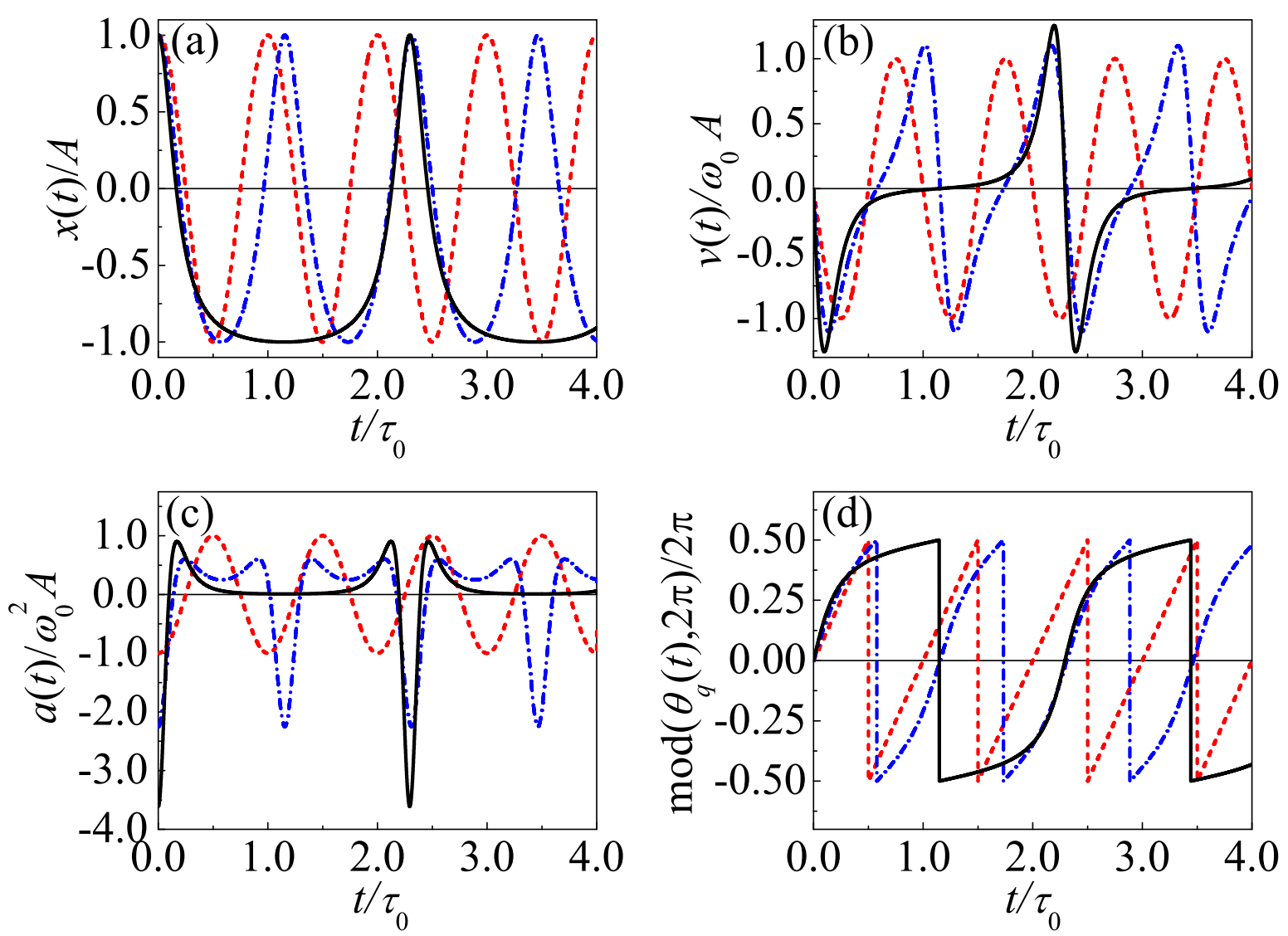

FIG. 1. (Color online) Temporal evolution of (a) position, (b) velocity, (c) acceleration and (d) phase for a PDM classical oscillator given by Eq. (19). $\gamma_{q} A=0$ (dashed red), 0.5 (dash-dotted blue), and 0.9 (solid black), $\left(\tau_{0}=2 \pi / \omega_{0}, \delta=0\right)$.

the absolute value of the speed gradually decreases and eventually the particle comes to rest at $t \rightarrow \infty$.

Equation (16) transforms the Hamiltonian $H(x, p)$ to the Morse oscillator Hamiltonian [40]

$$
K\left(x_{q}, p_{q}\right)=\frac{p_{q}^{2}}{2 m_{0}}+W_{q}\left(e^{-\alpha_{q} x_{q}}-1\right)^{2},
$$

with the binding energy $W_{q} \equiv m_{0} \omega_{0}^{2} / 2 \gamma_{q}^{2}$, and $\alpha_{q} \equiv-\gamma_{q}$ is a parameter that controls the anharmonicity of the potential $\left(\gamma_{q}^{2} A^{2}=E / W_{q}\right)$. Therefore, the classical canonical transformation Eq. (16) maps a system with PDM given by Eq. (19) subject to a quadratic potential in a phase space $(x, p)$ into a system of constant mass subject to a Morse potential in the deformed phase space $\left(x_{q}, p_{q}\right)$. Since $\gamma_{q}^{2} A^{2}=E / W_{q}$, the particle presents closed curves in the phase space $\left(x_{q}, p_{q}\right)$ for $E<W_{q}$ and an open curves for $E>W_{q}$. Figure 3 shows the phase 

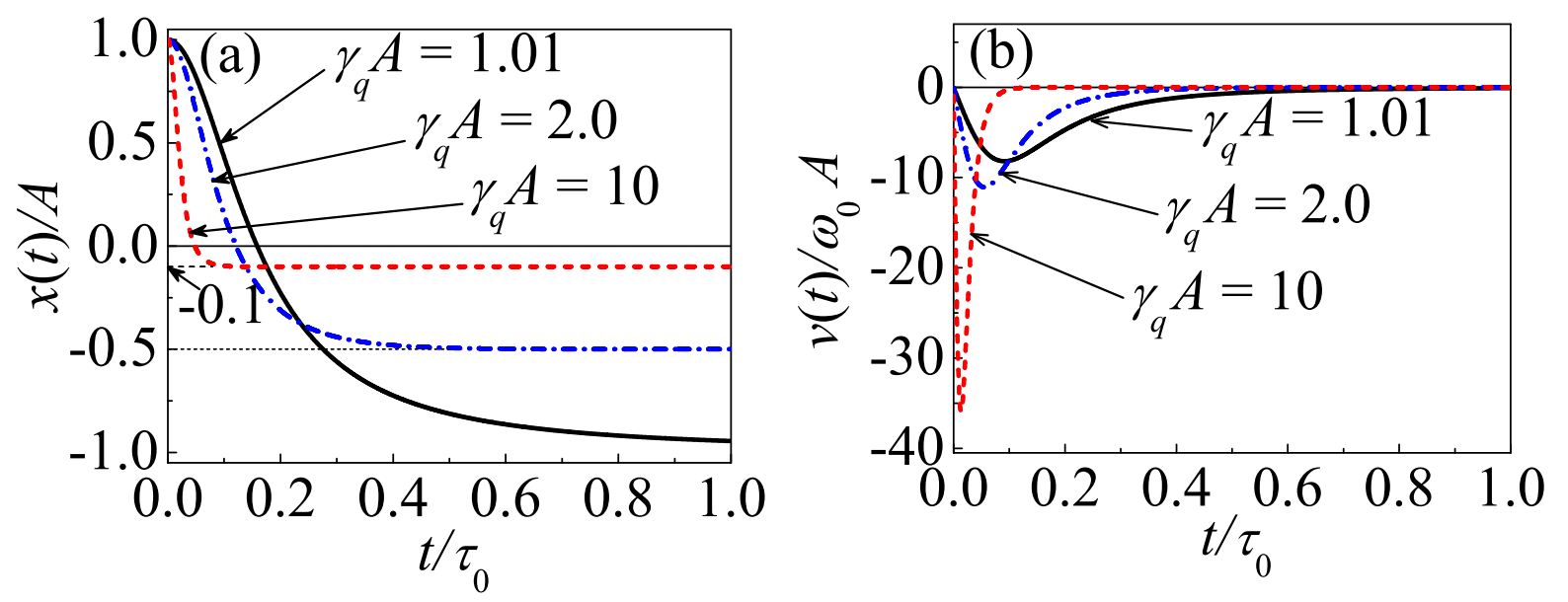

FIG. 2. (Color online) Temporal evolution of position and velocity for a PDM classical oscillator (Eq. (19) ). $\gamma_{q} A=1.01$ (solid black), 2.0 (dash-dotted blue), and 10 (dashed red).

spaces $(x, p)$ and $\left(x_{q}, p_{q}\right)$ for $\gamma_{q} A<1$ (closed orbits) and $\gamma_{q} A>1$ (open orbits). The confined position $\left(x_{\min }<x \leq A\right)$ and divergent momentum $(-\infty<p<\infty)$ for the problem with $\gamma_{q} A>1$ is turned into unconfined deformed position $\left(-\infty<x_{q}<x_{q, \max }=\gamma_{q}^{-1} \ln \left(1+\gamma_{q} A\right)\right)$ and bounded deformed momentum $\left(-p_{q, \max }<p_{q}<p_{q, \max }\right.$, with $\left.p_{q, \max }=m_{0} \omega_{0} A \sqrt{1-\frac{1}{\gamma_{q}^{2} A^{2}}}\right)$. Figure 4 brings some instances of Lissajous curves for two oscillators.

The probability $P_{\text {classic }}(x) d x \propto d x / v$ to find the particle with position between $x$ and $x+d x$ is

$$
P_{\text {classic }}(x) d x=\frac{\sqrt{1-\gamma_{q}^{2} A^{2}}}{\pi\left(1+\gamma_{q} x\right) \sqrt{A^{2}-x^{2}}} d x .
$$

The first and second moments of position and momentum are

$$
\begin{gathered}
\frac{\bar{x}}{A}=-\frac{1-\sqrt{1-\gamma_{q}^{2} A^{2}}}{\gamma_{q} A} \\
\frac{\overline{x^{2}}}{A^{2}}=\frac{1-\sqrt{1-\gamma_{q}^{2} A^{2}}}{\gamma_{q}^{2} A^{2}} \\
\bar{p}=0 \\
\overline{p^{2}}=\frac{m_{0}^{2} \omega_{0}^{2} A^{2}}{2\left(1-\gamma_{q}^{2} A^{2}\right)}
\end{gathered}
$$

The virial theorem is only valid for the usual case $\gamma_{q}=0$ since (see Eq. (48b), with $\bar{T}=$ $E-\bar{V}): \bar{T}=\sqrt{1-\gamma_{q}^{2} A^{2}} \bar{V}$ 

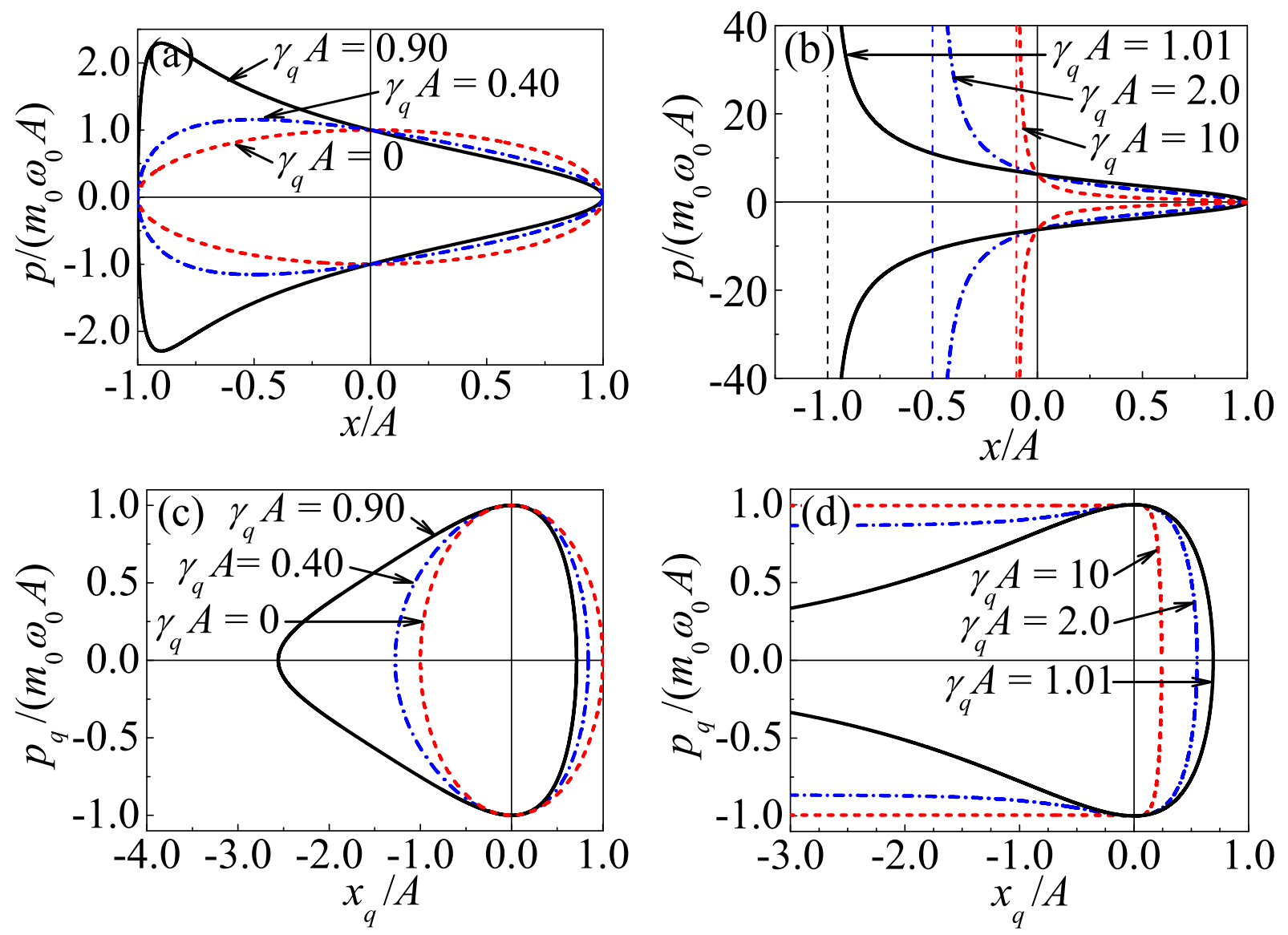

FIG. 3. (Color online) Phase spaces for a classical oscillator with $m(x)$ given by Eq. (19). Upper line: undeformed canonical coordinates $(x, p)$. Bottom line: deformed canonical coordinates $\left(x_{q}, p_{q}\right)$. Left column: closed orbits. Right column: open orbits.

The use of WKB approximation is an alternative and simple way to obtain the energy levels of a quantum system through its classical analogue. This procedure leads to

$$
\begin{aligned}
\left(n+\frac{1}{2}\right) \pi \hbar & =\frac{1}{2 \pi} \int_{-A}^{A} p d x=\frac{m \omega_{0}}{2 \pi} \int_{-A}^{A} \frac{\sqrt{A^{2}-x^{2}}}{1+\gamma_{q} x} d x \\
& =\frac{m \omega_{0} A^{2}}{4 \pi} \int_{0}^{2 \pi} \frac{\sin ^{2} \theta_{q}}{1+\gamma_{q} A \cos \theta_{q}} d \theta_{q}
\end{aligned}
$$

with $n$ integer. Solving the above equation for $A^{2}=2 E_{n} / m_{0} \omega_{0}^{2}$, we arrive at

$$
E_{n}=\omega_{0} \hbar\left(n+\frac{1}{2}\right)\left[1-\frac{\gamma_{q}^{2} x_{0}^{2}}{2}\left(n+\frac{1}{2}\right)\right],
$$

where $x_{0}^{2}=\hbar / m_{0} \omega_{0}$. Ref. [9] obtained the same result by using a non Hermitian linear momentum, Eq. (2). The energy levels of the quantum harmonic oscillator with PDM given by Eq. (19) are identical to those of a constant mass particle in a constant Morse potential, since these two systems may be mapped into one another by the canonical transform (16). 

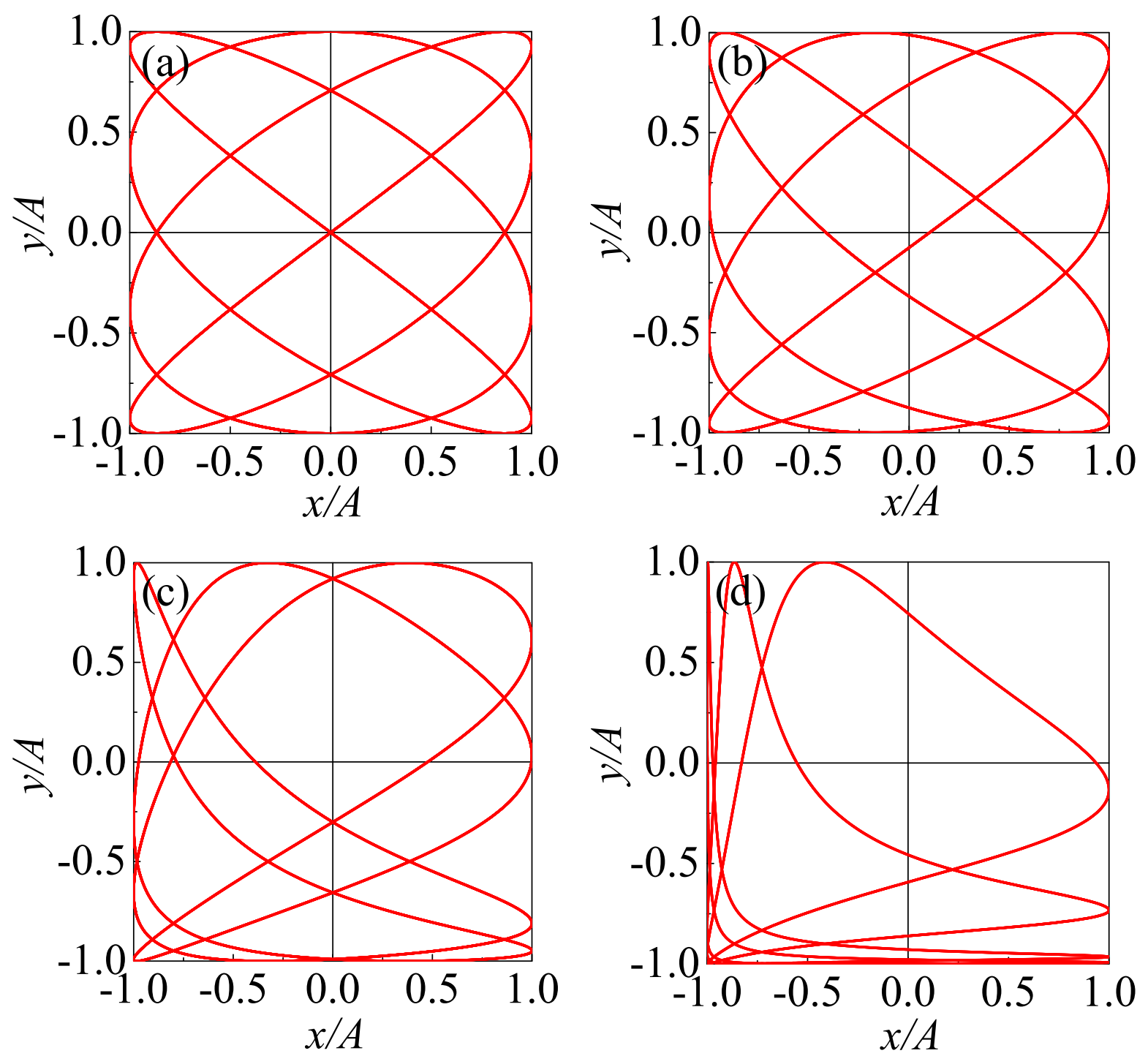

FIG. 4. (Color online) Lissajous figures for two deformed oscillators with phase difference $\delta_{y}-\delta_{x}=$ $\pi / 2$ and $\omega_{y} / \omega_{x}=4 / 3$ with $\gamma_{q} A$ equal to (a) 0 , (b) 0.3 , (c) 0.5 , and (d) 0.9 .

Figure 5 shows the Morse potential $V\left(x_{q}\right)=W_{q}\left(e^{-\alpha_{q} x_{q}}-1\right)^{2}$ for different values of $\gamma_{q} A$, as well as energy levels of bound states.

\section{QUANTUM HARMONIC OSCILLATOR WITH POSITION-DEPENDENT} MASS

The Hamiltonian operator for the PDM oscillator is

$$
\hat{H}=-\frac{\hbar^{2}}{2}\left\{[m(\hat{x})]^{-1 / 4} \frac{d}{d x}[m(\hat{x})]^{-1 / 2} \frac{d}{d x}[m(\hat{x})]^{-1 / 4}\right\}+\frac{1}{2} m_{0} \omega_{0}^{2} \hat{x}^{2},
$$



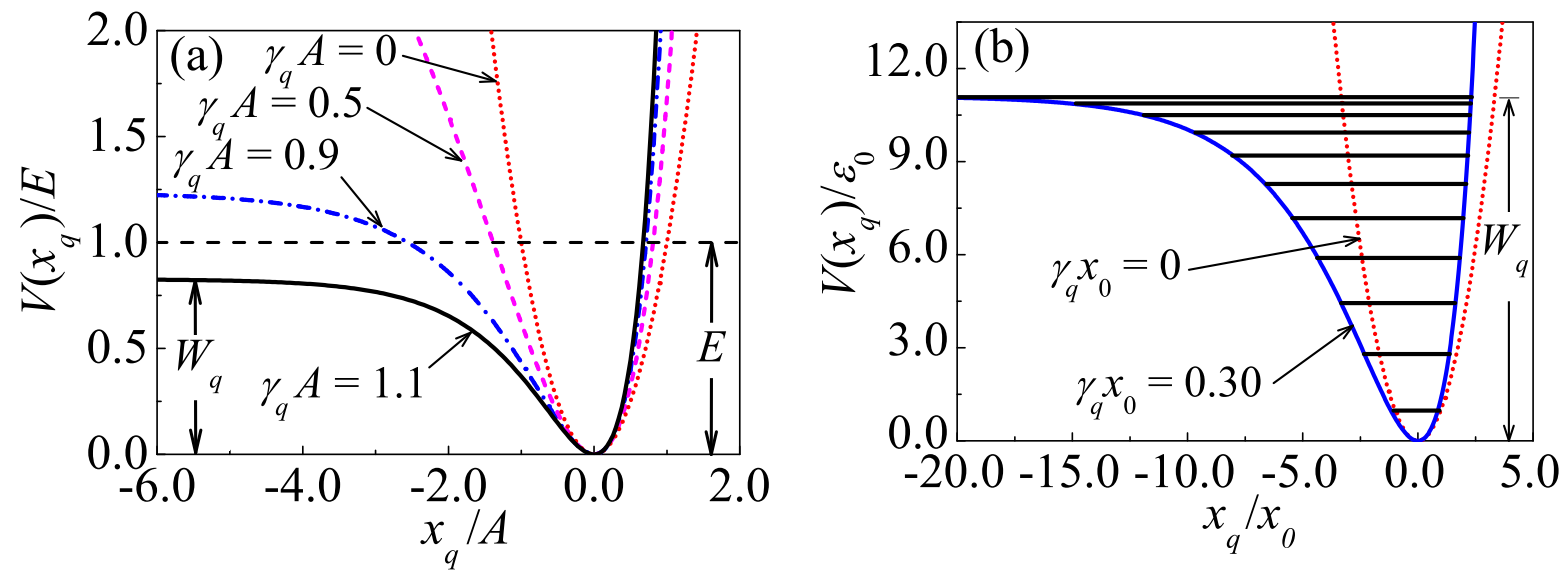

FIG. 5. (Color online) (a) Potentials in the phase space and $\left(x_{q}, p_{q}\right)$ for values of $\gamma_{q} A=0$ (dotted red), 0.5 (dashed magenta), 0.9 (dash-dotted blue), and 1.1 (solid black). (b) Energy levels of the bound states for $\gamma_{q} x_{0}=0.3$, with $\varepsilon_{0}=\hbar \omega_{0} / 2$. The usual case $\gamma_{q} x_{0}=0$ is shown for comparison.

and its associated time independent Schrödinger equation at basis $\{|x\rangle\}$ is

$$
-\frac{\hbar^{2}\left(1+\gamma_{q} x\right)^{2}}{2 m_{0}} \frac{d^{2} \psi(x)}{d x^{2}}-\frac{\hbar^{2} \gamma_{q}\left(1+\gamma_{q} x\right)}{m_{0}} \frac{d \psi(x)}{d x}-\frac{\hbar^{2} \gamma_{q}^{2}}{8 m_{0}} \psi(x)+\frac{1}{2} m_{0} \omega_{0}^{2} x^{2} \psi(x)=E \psi(x),
$$

where $\Psi(x, t)=\psi(x) e^{-i E t / \hbar}$ and $\langle x \mid \alpha\rangle=\psi(x)$. The the canonical transformation given by Eqs. (3) and (15) leads to the time independent Schrödinger equation at basis $\{|x\rangle\}$ for a particle with constant mass $m_{0}$ submitted to the Morse potential:

$$
-\frac{\hbar^{2}}{2 m_{0}} \frac{d^{2} \varphi\left(x_{q}\right)}{d x_{q}^{2}}+W_{q}\left(e^{-\alpha_{q} x_{q}}-1\right)^{2} \varphi\left(x_{q}\right)=E \varphi\left(x_{q}\right)
$$

The corresponding time independent deformed Schrödinger equation for the state functions $\varphi_{q}(x)=\varphi\left(x_{q}(x)\right)=\sqrt{1+\gamma_{q} x} \psi(x)$ is

$$
-\frac{\hbar^{2}}{2 m_{0}} D_{\gamma_{q}}^{2} \varphi_{q}(x)+\frac{1}{2} m_{0} \omega_{0}^{2} x^{2} \varphi_{q}(x)=E \varphi_{q}(x) .
$$

The solution of the above equations lead to the following wave functions:

$$
\psi_{n}(x)=\frac{A_{n}}{\sqrt{1+\gamma_{q} x}} e^{-d\left(1+\gamma_{q} x\right)}\left[2 d\left(1+\gamma_{q} x\right)\right]^{b / 2} L_{n}^{(b)}\left(2 d\left(1+\gamma_{q} x\right)\right),
$$

where $d=1 / \gamma_{q}^{2} x_{0}^{2}, b=2 d-1-2 n>0, A_{n}^{2}=b \gamma_{q} n ! /(n+b) !$ and $L_{n}^{(b)}$ are associated Laguerre polynomials. The energy levels are also given by Eq. (50). Figure 6 shows the wave function and density probability. Figure 7 illustrates that for large quantum numbers, here exemplified with $n=10$, the average of the quantum probability density $\rho_{n}(x)=\left|\psi_{n}(x)\right|^{2}$ 
approaches to the classical one given by Eq. (47) with amplitude $A=a_{q, n}$, where

$$
a_{q, n}^{2}=\frac{2 E_{n}}{m_{0} \omega_{0}^{2}}=x_{0}^{2}(2 n+1)\left[1-\frac{\gamma_{q}^{2} x_{0}^{2}}{4}(2 n+1)\right] \text {. }
$$

The deformed translation operator (1) can be extended to quantum systems with higher spatial dimensions through $\hat{T}_{q}(\vec{\varepsilon})|\vec{r}\rangle \equiv\left|x+\left(1+\gamma_{q} x\right) \varepsilon_{x}, y+\left(1+\gamma_{q} y\right) \varepsilon_{y}, z+\left(1+\gamma_{q} z\right) \varepsilon_{z}\right\rangle$, with $|\vec{r}\rangle=|x, y, z\rangle=|x\rangle \otimes|y\rangle \otimes|z\rangle$. The linear momentum operator, Eq. (3), is straightforwardly rewritten as $\vec{p}_{q}=p_{q, x} \vec{\imath}+p_{q, y} \vec{\jmath}+p_{q, z} \vec{k}$, with

$$
\begin{aligned}
\hat{p}_{q, x} & =\frac{\left(\hat{1}+\gamma_{q} \hat{x}\right) \hat{p}_{x}}{2}+\frac{\hat{p}_{x}\left(\hat{1}+\gamma_{q} \hat{x}\right)}{2}, \\
\hat{p}_{q, y} & =\frac{\left(\hat{1}+\gamma_{q} \hat{y}\right) \hat{p}_{y}}{2}+\frac{\hat{p}_{y}\left(\hat{1}+\gamma_{q} \hat{y}\right)}{2} \\
\hat{p}_{q, z} & =\frac{\left(\hat{1}+\gamma_{q} \hat{z}\right) \hat{p}_{z}}{2}+\frac{\hat{p}_{z}\left(\hat{1}+\gamma_{q} \hat{z}\right)}{2} .
\end{aligned}
$$

The canonical transformation (16) extended for three dimensions becomes $(\vec{p}, \vec{r}) \rightarrow\left(\vec{p}_{q}, \vec{r}_{q}\right)$ where $\vec{r}_{q}=\gamma_{q}^{-1}\left[\ln \left(1+\gamma_{q} x\right) \vec{i}+\ln \left(1+\gamma_{q} y\right) \vec{j}+\ln \left(1+\gamma_{q} z\right) \vec{k}\right]$.

Figure 8 shows probability densities $\rho_{n_{1}, n_{2}}(x, y)=\left|\psi_{n_{1}}(x) \psi_{n_{2}}(y)\right|^{2}$ for the two-dimensional case, where the asymmetric effect due to the PDM can be seen. The optical analog of this two-dimensional oscillator is related to the propagation of Gaussian beams in nonhomogeneous media [41].

The expectation values of $\langle\hat{x}\rangle,\left\langle\hat{x}^{2}\right\rangle,\langle\hat{p}\rangle$, and $\left\langle\hat{p}^{2}\right\rangle$ are

$$
\begin{gathered}
\langle\hat{x}\rangle=-\frac{\gamma_{q} \hbar}{m_{0} \omega}\left(n+\frac{1}{2}\right), \\
\left\langle\hat{x}^{2}\right\rangle=\frac{\hbar}{m_{0} \omega}\left(n+\frac{1}{2}\right), \\
\langle\hat{p}\rangle=0 \\
\left\langle\hat{p}^{2}\right\rangle=m_{0} \omega \hbar \frac{\left(n+\frac{1}{2}\right)-\frac{\gamma_{q}^{2} x_{0}^{2}}{2}\left(n^{2}+n-1\right)}{\left[1-\gamma_{q}^{2} x_{0}^{2}\left(n+\frac{1}{2}\right)\right]^{2}-\left(\gamma_{q} x_{0}\right)^{4}} .
\end{gathered}
$$

We can clearly see that in the limit $\gamma_{q} x_{0} \rightarrow 0$, the usual cases $\langle\hat{x}\rangle=0$ and $\left\langle\hat{p}^{2}\right\rangle=$ $m_{0} \omega \hbar\left(n+\frac{1}{2}\right)$ are recovered. Since $b / 2 d=\sqrt{1-\gamma_{q}^{2} a_{q, n}^{2}}$, Eqs. (58a), (58b) and (58d) can be rewritten as

$$
\begin{aligned}
\frac{\langle\hat{x}\rangle}{a_{q, n}} & =-\frac{1-\sqrt{1-\gamma_{q}^{2} a_{q, n}^{2}}}{\gamma_{q} a_{q, n}}, \\
\frac{\left\langle\hat{x}^{2}\right\rangle}{a_{q, n}^{2}} & =\frac{1-\sqrt{1-\gamma_{q}^{2} a_{q, n}^{2}}}{\gamma_{q}^{2} a_{q, n}^{2}}
\end{aligned}
$$



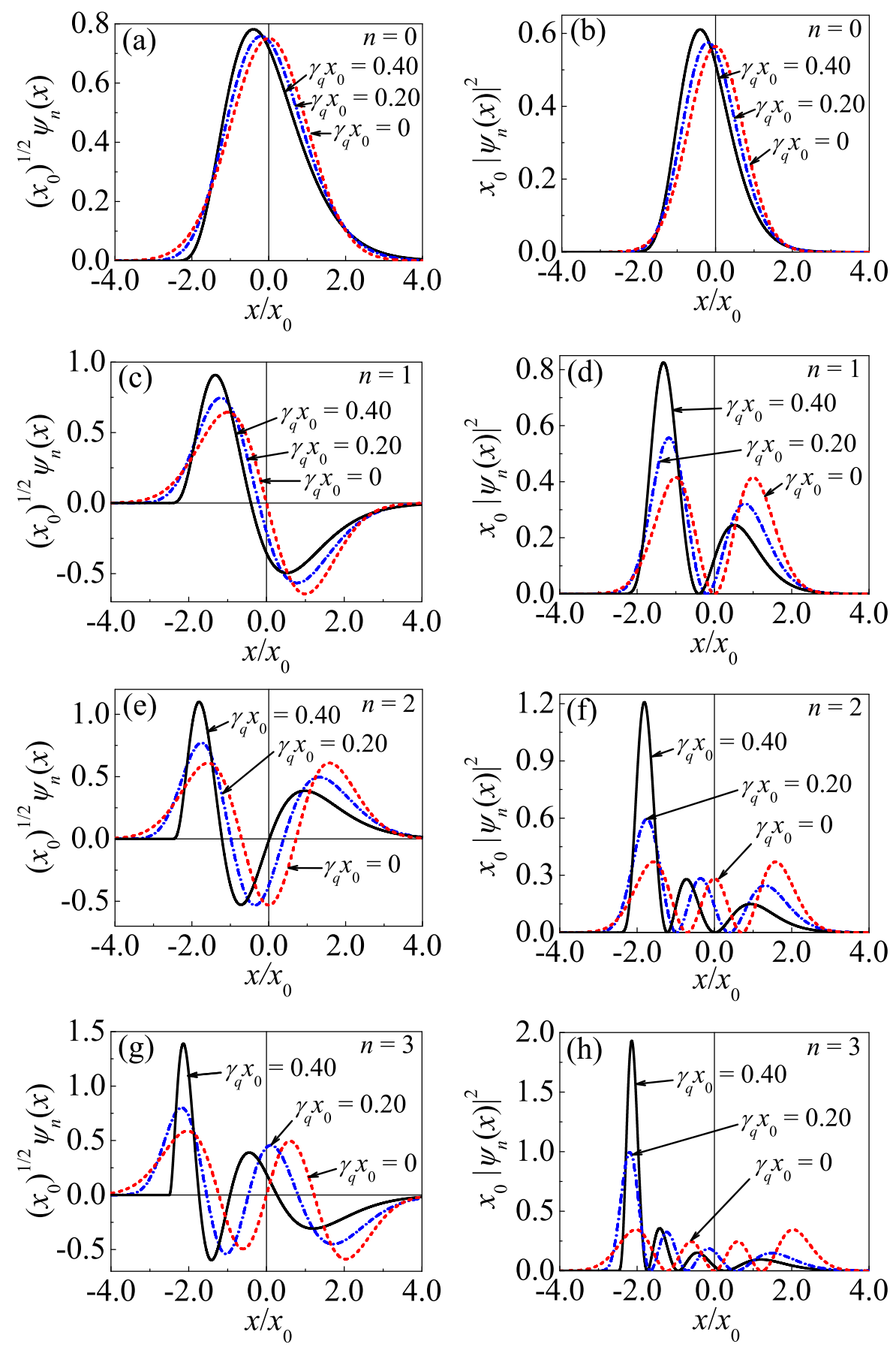

FIG. 6. (Color online) Wave functions $\psi_{n}(x)$ (left column) and probability densities $\left|\psi_{n}(x)\right|^{2}$ (right column) for a particle with PDM according to Eq. (19) under a quadratic potential for different values of $\gamma_{q} x_{0}$ (indicated, usual case $\gamma_{q} x_{0}=0$ is shown, for comparison). (a) and (b): $n=0$ (ground state), (c) and (d): $n=1$ (first excited state), (e) and (f): $n=2$ (second excited state). (g) and (h): $n=3$ (third excited state). 


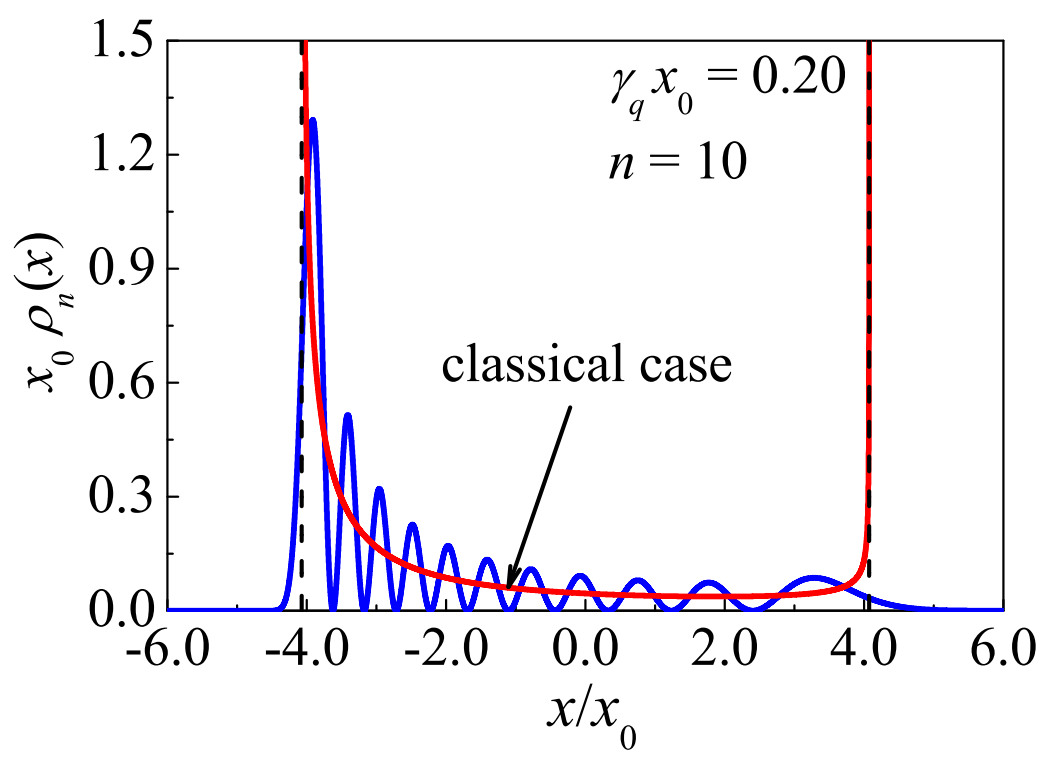

FIG. 7. (Color online) Probability density for a PDM (Eq. (19)) in a harmonic potential for $x_{0} \gamma_{q}=0.20$ and $n=10$. The classical case (Eq. (47)) is represented for comparison.

$$
\left\langle\hat{p}^{2}\right\rangle=\frac{m_{0}^{2} \omega_{0}^{2}}{2}\left(\frac{a_{q, n}^{2}+\frac{3}{4} \gamma_{q}^{2} x_{0}^{4}}{1-\gamma_{q}^{2} a_{q, n}^{2}-\gamma_{q}^{4} x_{0}^{4}}\right) .
$$

According to the principle of correspondence, in the limit of large quantum numbers (or $\hbar \rightarrow 0$ ), we have $E_{n} \rightarrow E$ and $a_{q, n} \rightarrow A$, and consequently Eqs. (59) coincide with Eqs. (48). Similarly to the classic case, one obtains $\langle\hat{T}\rangle=\sqrt{1-\gamma_{q}^{2} a_{q, n}^{2}}\langle\hat{V}\rangle$.

Figure 9 shows the uncertainty relation for this oscillator. $\Delta x \Delta p \geq \hbar / 2$, since that the operators $\hat{x}$ and $\hat{p}$ are canonically conjugate and Hermitian.

\section{CONCLUSIONS}

We revisit the problem of a particle with position-dependent mass introduced in [9], where we use the Hermitian deformed linear momentum operator $\hat{p}_{q}$, instead of the originally proposed non-Hermitian operator. The definition of a deformed space operator $\hat{x}_{q}=\xi \ln \left[\exp _{q}(\hat{x} / \xi)\right]($ Eq. (15) $)$ ) establishes the equivalence of the PDM particle in an ordinary phase space to a constant mass particle in a deformed phase space. Particularly, the PDM $m(x)=m_{0} /\left(1+\gamma_{q} x\right)^{2}$ (Eq. (19) $)$ in an ordinary space submitted to a quadratic potential is transformed into a constant mass in a deformed space submitted to the Morse potential. Since the operators $\hat{x}_{q}$ and $\hat{p}_{q}$ are Hermitian and canonically conjugated, the 

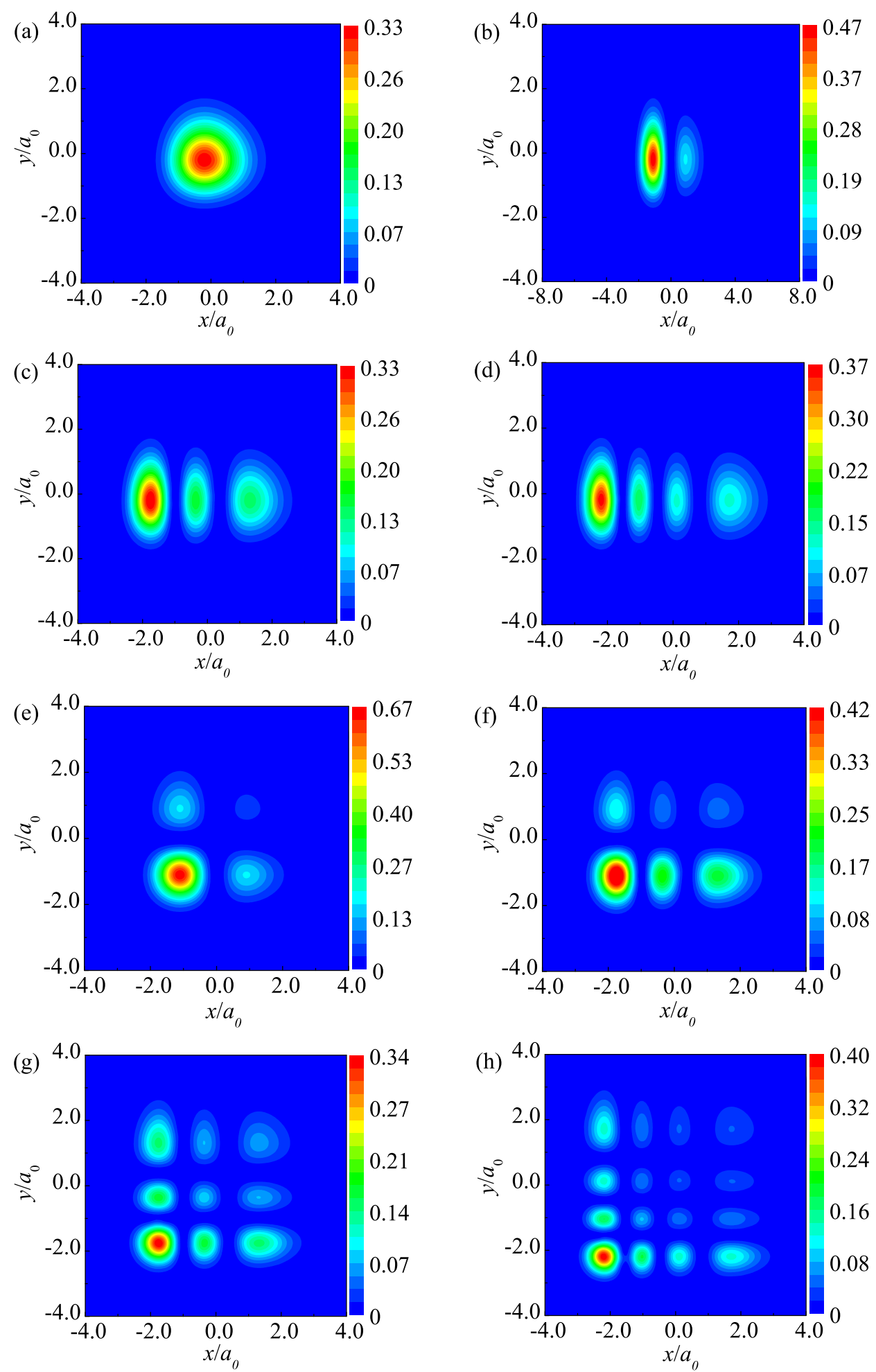

FIG. 8. (Color online) Probability densities $\rho_{n_{1}, n_{2}}(x, y)=\left|\psi_{n_{1}}(x) \psi_{n_{2}}(y)\right|^{2}$ (in units of $a_{0}^{-2}$ ) for $\gamma_{q} a_{0}=0.2$. (a) $\left(n_{1}, n_{2}\right)=(0,0),(\mathrm{b})(1,0),(\mathrm{c})(2,0),(\mathrm{d})(3,0),(\mathrm{e})(1,1),(\mathrm{f})(2,1),(\mathrm{g})(2,2),(\mathrm{h})$ $(3,3)$. Color scale ranges from blue (low probabilities) to red (high probabilities). 

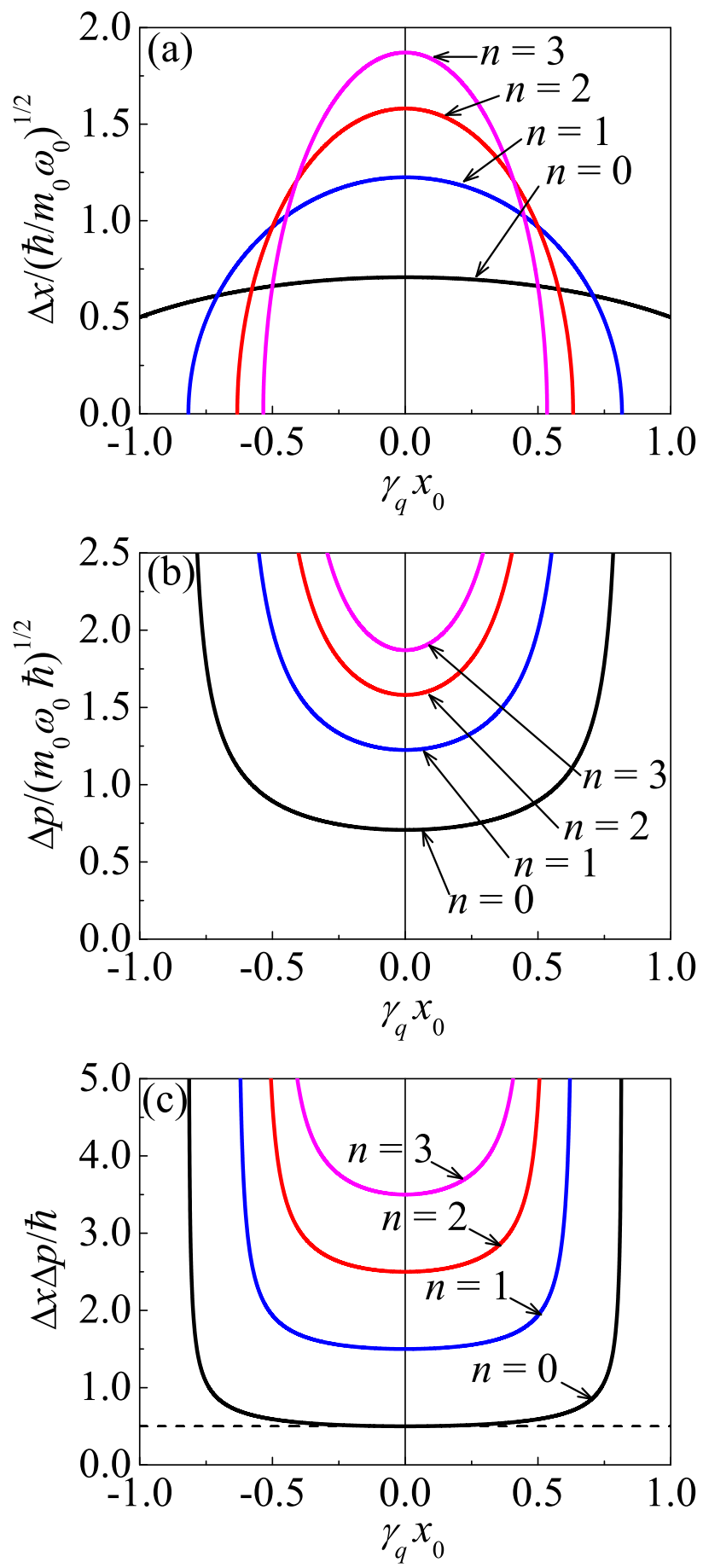

FIG. 9. (Color online) Uncertainty of (a) position $(\Delta x)$, (b) linear momentum $(\Delta p)$, (c) $\Delta x \Delta p$, for the first quantum numbers. The usual case $\Delta x \Delta p=\left(n+\frac{1}{2}\right) \hbar$ is recovered for $\gamma_{q} x_{0} \rightarrow 0$.

classical and quantum formalisms are coherently connected, and the uncertainty and correspondence principles are consistently followed. The introduction of deformed derivatives allows the equations of motion to be written with a formal similarity to the usual ones: 
the quantum case uses the q-derivative, Eq. (6) (see Eq. (34)), while the classical case uses the dual q-derivative, Eq. (7) (see Eq. (22)). The specific PDM we have considered here, Eq. (19), is associated with the deformed mathematical framework addressed in Sec. III, Investigations of the deformed oscillator through factorization methods in quantum mechanics,

like supersymmetry [6, 7] and generalized Heisenberg algebras [42, 43], and how the operators that emerge from these formulations can be rewritten in terms of $q$-deformed functions and derivatives are interesting additional developments. The equivalence between the use of deformed variables and deformed functions is already present in the works of Kaniadakis, e.g., Eq. (50) of [44] and Eq. (2.1) of [45], though in a different context. Other possible PDM functions may be connected to different deformations of space, and consequently to different derivatives, and ultimately, deformed equations of motion. Investigations on these possible unexpected connections are stimulating.

\section{ACKNOWLEDGMENTS}

We thank M. A. Rego-Monteiro and F. F. dos Santos for fruitful discussions. This work was partially supported by FAPESB (Brazilian agency), through the program PRONEX.

[1] O. von Roos, Phys. Rev. B 27, 7547 (1983).

[2] N. Aquino, G. Campoy, and H. Yee-Madeira, Chem. Phys. Lett. 296, 111 (1998).

[3] F. Arias de Saavedra, J. Boronat, A. Polls, and A. Fabrocini, Phys. Rev. B 50, 4248 (1994).

[4] R. Khordad, Indian J. Phys. 86, 513 (2012).

[5] H. A. Bethe, Phys. Rev. Lett. 56, 1305 (1986).

[6] A. R. Plastino, A. Rigo, M. Casas, F. Garcias, and A. Plastino, Phys. Rev. A 60, 4318 (1999).

[7] N. Amir and S. Iqbal, J. Math. Phys. 57, 062105 (2016).

[8] R. N. Costa Filho, M. P. Almeida, G. A. Farias, and J. S. Andrade, Jr., Phys. Rev. A 84, 050102(R) (2011).

[9] R. N. Costa Filho, G. Alencar, B.-S. Skagerstam, and J. S. Andrade, Jr., Europhys. Lett. 101, 10009 (2013). 
[10] S. H. Mazharimousavi, Phys. Rev. A 85, 034102 (2012); Phys. Rev. A 89, 049904(E) (2014) (erratum).

[11] B. G. da Costa and E. P. Borges, J. Math. Phys. 55, 062105 (2014).

[12] L. Nivanen, A. Le Méhauté, and Q. A. Wang, Rep. Math. Phys. 52, 437 (2003).

[13] E. P. Borges, Physica A 340, 95 (2004).

[14] M. A. Rego-Monteiro and F. D. Nobre, Phys. Rev. A 88, 032105 (2013).

[15] E. G. Barbagiovanni, D. J. Lockwood, N. L. Rowell, R. N. Costa Filho, I. Berbezier, G. Amiard, L. Favre, A. Ronda, M. Faustini, and D. Grosso, J. Appl. Phys. 115, 044311 (2014).

[16] E. G. Barbagiovanni and R. N. Costa Filho, Physica E 63, 14 (2014).

[17] M. Vubangsi, M. Tchoffo, and L. C. Fai, Physica Scripta 89, 025101 (2014).

[18] M. Vubangsi, M. Tchoffo, and L. C. Fai, Eur. Phys. J. Plus 129, 105 (2014).

[19] M. Tchoffo M. Vubangsi, and L. C. Fai, Physica Scripta 89, 105201 (2014).

[20] A. Arda and R. Sever, Few-Body Systems 56(10), 697 (2015).

[21] F. D. Nobre, M. A. Rego-Monteiro, and C. Tsallis, Phys. Rev. Lett. 106, 140601 (2011).

[22] F. D. Nobre, M. A. Rego-Monteiro, and C. Tsallis, Europhys. Lett. 97, 41001 (2012).

[23] A. R. Plastino and C. Tsallis, J. Math. Phys. 54, 041505 (2013).

[24] M. A. Rego-Monteiro and F. D. Nobre, J. Math. Phys. 54, 103302 (2013).

[25] A.R. Plastino, A. M. C. Souza, F. D. Nobre, and C. Tsallis, Phys. Rev. A 90, 062134 (2014).

[26] F. Pennini, A. R. Plastino, and A. Plastino, Physica A 403, 195 (2014).

[27] T. Bountis and F.D. Nobre, J. Math. Phys. 57, 082106 (2016).

[28] D.J. Zamora, M.C. Rocca, A. Plastino, and G.L. Ferri, Entropy 19, 21 (2017).

[29] A. Macfarlane, J. Phys. A: Math. Gen. 22, 4581 (1989).

[30] L. Biedenharn, J. Phys. A: Math. Gen. 22, L873 (1989).

[31] A. Lavagno, J. Phys. A: Math. Theor. 41, 244014 (2008).

[32] A. Lavagno and G. Gervino, Journal of Physics: Conference Series 174, 012071 (2009).

[33] A. Lavagno, Reports on Mathematical Physics 64(1-2), 79 (2009).

[34] E. P. Borges, J. Phys. A: Math. Gen. 31, 5281 (1998).

[35] C. Tsallis, Quimica Nova 17, 468 (1994).

[36] C. Tsallis, J. Stat. Phys. 52, 479 (1988).

[37] C. Tsallis, Introduction to Nonextensive Statistical Mechanics - Approaching a Complex World (Springer, New York, 2009). 
[38] S. Cruz y Cruz and O. Rosas-Ortiz, SIGMA 9, 004 (2013).

[39] S. Cruz y Cruz and O. Rosas-Ortiz, J. Phys. A: Math. Theor. 42, 185205 (2009).

[40] P. M. Morse, Phys. Rev. 34, 57 (1929).

[41] A. Yariv, Quantum eletronics (John Wiley \& Sons, New York, 1988).

[42] E. M. F. Curado, M.A. Rego-Monteiro, J. Phys. A: Math. Gen. 34, 3253 (2001).

[43] E. M. F. Curado, M. A. Rego-Monteiro, and L. M. Rodrigues, Phys. Rev. A, 87(5), 052120 (2013).

[44] G. Kaniadakis, Physica A 296, 405 (2001).

[45] G. Kaniadakis, Phys. Rev. E 66, 056125 (2002). 